Trauma Berufskrankh 2018 20 (Suppl 1):S12-S16 https://doi.org/10.1007/s10039-017-0338-7 Online publiziert: 13. Dezember 2017 (c) Springer Medizin Verlag GmbH, ein Teil von Springer Nature 2017

CrossMark

\section{K. Großer}

Klinik für Kinderchirurgie \& Kinderurologie, Zentrum für schwerbrandverletzte Kinder, Frau-Mutter-KindZentrum Erfurt, Helios-Klinikum GmbH Erfurt, Erfurt, Deutschland

\section{Behandlung der kindlichen Unterarmschaftfraktur}

\section{Was geht noch konservativ?}

Kindliche Unterarmfrakturen sind mit über $30 \%$ häufig. Aber nur $25 \%$ davon betreffen die Diaphyse selbst. Dabei nimmt die Frakturhäufigkeit von proximal nach distal zu [1]. Als Unfallmechanismus ist eine indirekte Gewalteinwirkung auf den Unterarmschaft typisch. Dabei sind Stürze der Kinder auf den gestreckten Arm sehr häufig. Dagegen tritt ein direktes Trauma selten auf. Es kann sich dann um eine sog. Parier-Fraktur handeln, die als Hinweis auf eine Battered-child-Situation gelten kann [2].

In der Symptomatik imponiert häufig schon die sichtbare Fehlstellung mit der schmerzhaften Functio laesa. Nach

Tab. 1 Klassifikation der kindlichen Unterarmschaftfrakturen nach Slongo [3]

Frakturen des

Abschnitts 22

Isolierte Radiusfrak-

turen

Isolierte Ulnafraktu-

ren

Ulnafrakturen mit (Monteggia) und ohne Radiusköpfchenluxation

Unterarmfrakturen

A - Vollständige Frakturen (D4 oder D5) Quere Bruchformen

B - Grünholzfrakturen

Schräge Bruchformen

Biegungsfraktur

Periost und/oder Kortikalis stehen einseitig

Mit/ohne wesentliche Achsdeviation

Oft erhebliche Achsdeviation (D2) Sonderformen

Monteggia (D6)

Galeazzi (D7) der Beurteilung der Weichteilverhältnisse bezüglich Durchblutung, Sensibilität und Motorik besteht die radiologische Diagnostik in der Anfertigung von nativen Röntgenaufnahmen in 2 Ebenen als Standard (• Abb. 1). Dabei wird der Frakturtyp mit Ausmaß der Dislokation festgelegt. Die Klassifikation der kindlichen Unterarmschaftfrakturen ist in der - Tab. 1 dargestellt.

Um in der Therapie dieser Frakturen die Frage beantworten zu können: „Was geht noch konservativ?“, lohnt es, die Ziele dieser Frakturbehandlung für das Kind näher zu beleuchten. Das Ziel der Frakturbehandlung im Kindesalter ist zuerst, das weitere Wachstum nicht zu stören und somit einen funktionell intakten Bewegungsapparat am Wachstumsabschluss zu gewährleisten. Die praktische Erfahrung zeigt dabei, dass das gute

Tab. 2 Operationsindikation bei kindli-
chen Unterarmfrakturen
Instabile und irreponible Frakturen
(• Abb. 4)
Reponierbare, aber nicht retinierbare Fraktu-
ren
Sekundär dislozierte Frakturen mit Achsfeh-
ler $>10^{\circ}$
Pathologische Frakturen
Refrakturen
Frakturen im Rahmen von Polytrauma/
Multitrauma
Offene Frakturen mit/ohne Gefäß-/
Nervenverletzungen
Mehrfragmentfrakturen
Pseudarthrosen

Ergebnis im Kindesalter nicht immer auf das gewählte Therapieverfahren zurückzuführen ist. Und es heilen sogar kindliche Brüche ohne und manchmal sogar trotz ärztlicher Maßnahmen vollständig aus! Kritisch bleibt aber auch festzustellen, dass ein schlechtes Ergebnis nach einer operativen Stabilisierung immer auf einer fehlerhaften Osteosynthese basiert.

\section{Spontankorrekturen}

Das Potenzial der Spontankorrekturen am Unterarm gilt es somit gezielt auszunutzen.

Dabei läuft eine Spontankorrektur im Kindesalter immer zuverlässig ab. Nur das konkrete Ausmaß der Spontankorrektur ist individuell; diese ist abhängig von mehreren Faktoren. Wichtig sind das Alter des Kindes zum Unfallzeitpunkt

Tab. 3 Intraoperative ESIN (elastisch stabile intramedulläre Nagelung)-Tricks

Nagelstärke zwei Drittel Markraum nicht immer möglich

Immer mit dem schwerer zu reponierenden Knochen beginnen (oft Radius)

Kein Einklopfen des Nagels, sondern händisches Vordrehen mit Jakobs-Futter

Bevorzugte Implantation: Radius aszendierend, Ulna deszendierend

Feinreposition des Radius: bei schwerer Einstellung Nutzen des Prevot-Nagels als Joystick in Gegentechnik bei bereits ante portas liegendem Ulnanagel ( $\bullet$ Abb. 5)

Interponat: Miniinzision und Häkchenreposition

Mit Nagelenden abschließend Aufspannen der Membrana interossea

Vor Narkoseende: Weichteilmobilisierung in Pronation/Supination 


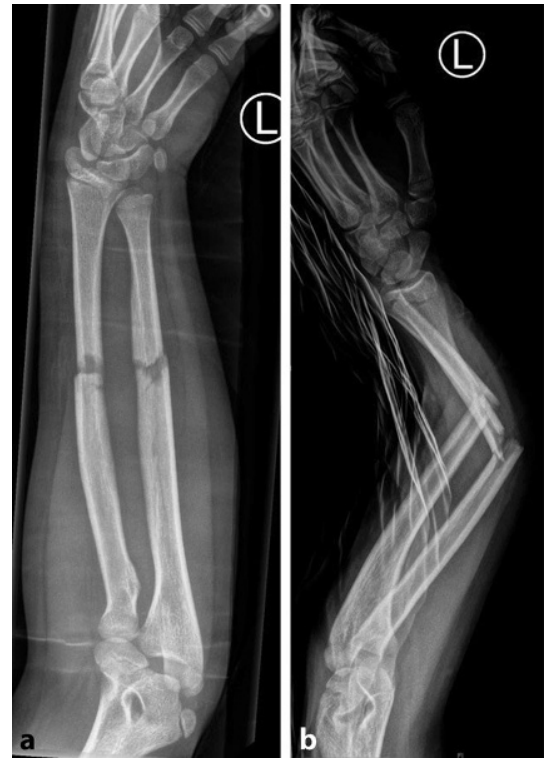

Abb. 1a, b $\Delta$ Röntgenunfallaufnahme mit Unterarmschaftfraktur mit deutlichem Achsknick in 2 Ebenen "seitlich angestellt". a a. p., b seitlich

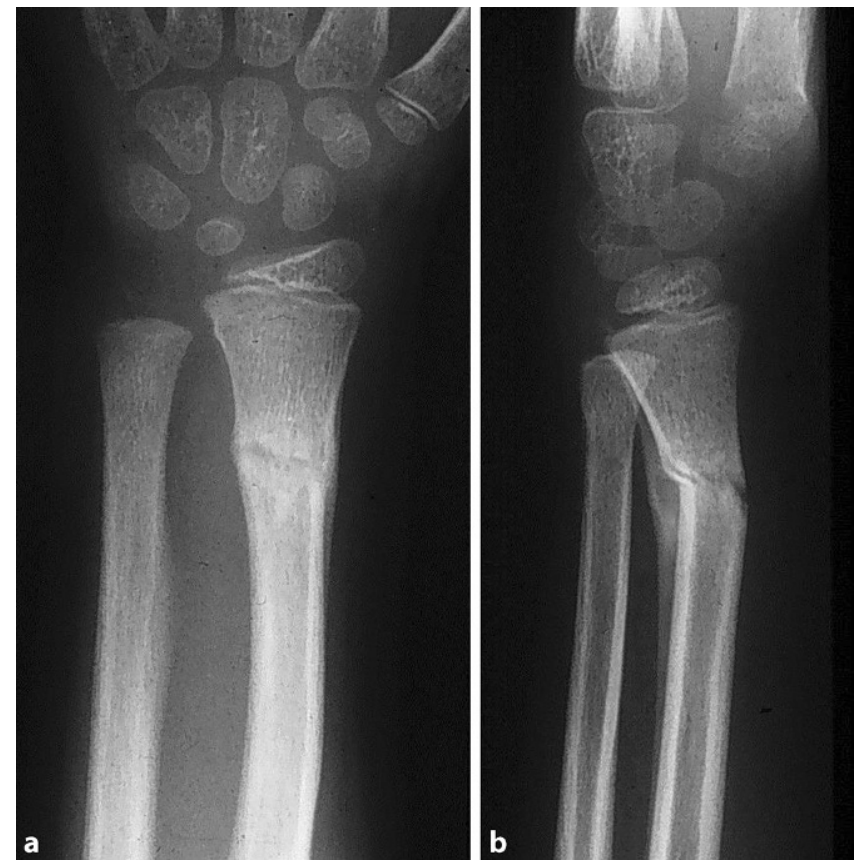

Abb. 2a, b $<$ Fallbeispiel 1: Remodelling distale Schaftfraktur (Grünholz, 7 Jahre, weiblich). a Röntgenaufnahme a.p.:achsengerechte Stellung, b Röntgenaufnahme seitlich:Wachstumsausgleich der Achsdeviation nach 3 Monaten
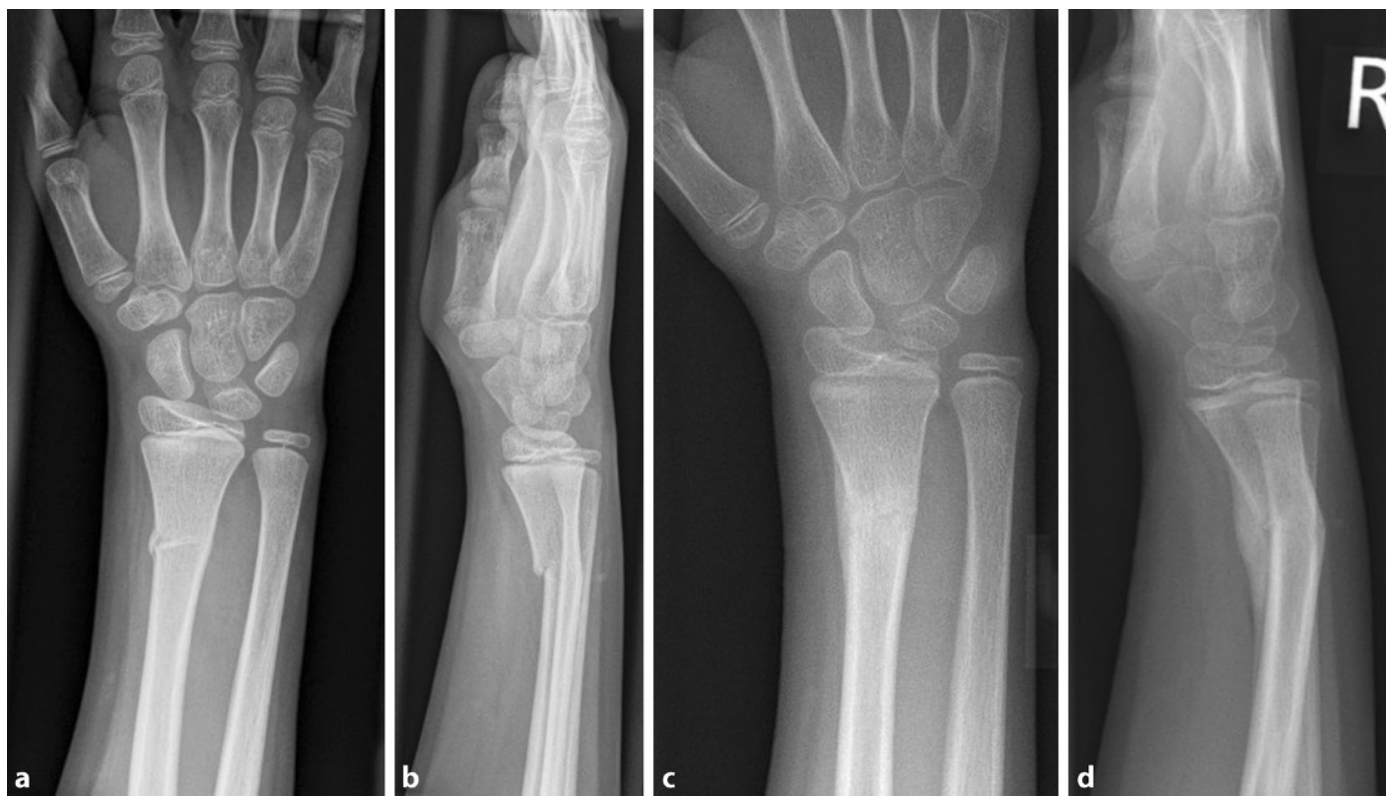

Abb. $3<$ Fallbeispiel 2:

Spontankorrekturam distalen Unterarmschaft (10 Jahre, männlich). a, b Röntgen am Unfalltag. c, d Röntgen nach 8 Wochen

und die konkrete Wachstumspotenz der nahe liegenden Fuge. Bedeutsam ist die Ebene, in der die Fraktur liegt - nicht jedoch der Frakturtyp [4].

Bekanntermaßen werden Achsfehler in der Bewegungsebene besser als in der Frontalebene ausgeglichen (• Abb. 2). Ein Varus wird besser als ein Valgus korrigiert. Die belastete untere Extremität gleicht vollständiger als die unbelastete obere Extremität aus. Und bei Rotationsfehlern gibt es praktisch keine relevante Korrektur [5]. Höchstes
Potenzial haben somit an den langen Röhrenknochen der proximale Humerus, der distale Unterarm und der distale Femur. Schlussfolgernd kann festgestellt werden, dass klassische Unterarmschaftfrakturen entgegen der weitverbreiteten Annahme eben keine nennenswerte Spontankorrektur haben! Einzige und dann auch praktisch relevante Ausnahmen sind die distalen Unterarmfrakturen im metaphysären Übergangsbereich, die ein vorzügliches Remodelling bis zum 10. Lebensjahr vollführen (• Abb. 3).

\section{Konservative Therapie}

In der Praxis werden anteilig die meisten diaphysären Unterarmfrakturen mit einer konservativen Therapie versehen. Dazu gehören alle undislozierten Frakturen, die in einem Oberarmgips versorgt werden. Außerdem sind dabei kleine Achsfehler bis $10^{\circ}$ in 1 Ebene mit gleicher Retention ohne Reposition mittels Gipsruhigstellung gut behandelbar. Wegen der Möglichkeit einer sekundären Dislokation sollte nach 1 Woche eine 
Röntgenkontrolle erfolgen, um dann zeitgerecht ggf. eine Gipskeilung vornehmen zu können.

Die Gipskeilung hat weiterhin ihre Berechtigung, muss aber bestimmte Regeln beachten. So sind a.p.-Achsfehler immer ungünstig für eine effektive Keilung. Soll eine Keilung von nur einem Knochen gegen den anderen unversehrten Knochen ausgeführt werden, so verhindert die Rigidität des intakten Knochens oft eine ausreichende Keilungswirkung.

Empfehlenswert ist eine Gipsretention je nach Alter von (3-)4-6 Wochen.

Jede weiter als $10^{\circ}$ dislozierte Fraktur sollte reponiert werden. Vollständig dislozierte Frakturen sind manchmal leicht zu reponieren, und eine vermeintlich leichte Reposition zeigt sich oft als irreponibel. Als Regel gilt bei jeder Reposition in Narkose beim Kind, dass die Möglichkeit der sofortigen Osteosynthese in gleicher Narkose gegeben sein muss!

\section{Unvollständige Frakturen der Diaphyse}

Die Grünholzfrakturen dislozieren immer ad axim, können aber nicht noch weiter verkürzen. Das vollständige Durchbrechen der Gegenkortikalis ist vorzunehmen, um eine Refraktur zu verhindern. Nachteil ist dabei die Umwandlung einer stabilen in eine instabile Fraktur. Als Alternative kann gelten, dass die Kortikalis nur mit Druck auf der Konvexseite gegengebogen wird und dies auch beim Anmodellieren des Gipses ausgeführt wird.

\section{Vollständige diaphysäre Frakturen}

Bei den vollständigen Unterarmfrakturen sind für die Indikation zur Reposition der Frakturverlauf und der Dislokationstyp entscheidend [6].

Bei Querfrakturen werden unter Relaxation (Anästhesie) mit Zug und Gegenzug die Fragmente aufeinander gestellt, und nicht selten ist sogar ein „Überbiegen“ des Unterarmes im Sinne des stattgehabten Frakturmechanismus sinnvoll. Eine Seitverschiebung bis auf fast Schaftbreite ist tolerabel, wenn dabei die Kortikalis günstig verhakt. Die Gipsretenti- on erfolgt in $90^{\circ}$-Ellenbogengelenk-Beugung und in Normal-Null-Stellung des Unterarmes oder besser noch in Supinationsstellung, um eine stabilisierende Wirkung der Membrana interossea zu erzielen. Zusätzliche Stabilität kann durch eine Gipsabflachung am Unterarm durch beide Handflächen des Gipsers erreicht werden, indem der Gips danach einer gedachten „Erdnussform“ im Gipsquerschnitt entspricht.

Bei Schrägfrakturen neigt der Radius zum Valgus und die Ulna zum Varus [7]. Bei kompletten Frakturen mit Zerreißung der Membrana interossea sind auch Rotationsfehler möglich. Die Reposition erfolgt zunächst wie bei Querfrakturen, ist aber weniger erfolgreich! Wenn keine vollständige Reposition möglich ist, dann ist eine konservative Therapie auch nicht mehr indiziert. Somit sollten dann die operative Reposition und Osteosynthese in gleicher Narkose erfolgen. Des Weiteren sollen bei Kindern keine Nachrepositionen vorkalkuliert werden! Wenn die Frakturmechanik jetzt keine Reposition zulässt, dann in der Regel später auch nicht.

Bei Ulnaschaftfrakturen muss zum Ausschluss einer Monteggia-Läsion immer nach einer begleitenden Radiusköpfchenluxation oder Radiushalsfraktur („Monteggia-like“) gesucht werden [8]. Gegebenenfalls ist eine Röntgenergänzung des Ellenbogengelenks in 2 Ebenen notwendig.

Eine weitere Besonderheit sind federnde Ulnaschaftfrakturen (sog. „bowing fractures“), die nicht selten unterschätzt werden und einer Osteosynthese bedürfen (bevorzugt elastisch stabile intramedulläre Nagelung [ESIN]).

\section{Operative Therapie der Unterarmfrakturen}

Zur operativen Therapie von diaphysären Unterarmfrakturen stehen mehrere Osteosyntheseverfahren zur Verfügung. Obwohl die ESIN die am häufigsten angewendete Osteosynthese für diese Frakturen darstellt, müssen die anderen Verfahren in bestimmten Situationen zusätzlich im Portfolio des Operateurs stehen.
Trauma Berufskrankh $2018 \cdot 20$ (Suppl 1):S12-S16 https://doi.org/10.1007/s10039-017-0338-7

○) Springer Medizin Verlag GmbH, ein Teil von Springer Nature 2017

\section{K. Großer}

\section{Behandlung der kindlichen Unterarmschaftfraktur. Was geht noch konservativ?}

\section{Zusammenfassung}

Alle nicht und gering dislozierten Unterarmschaftfrakturen sollten weiterhin mittels Oberarmgipsretention versorgt werden. Für die dislozierte diaphysäre Unterarmschaftfraktur jedoch ist die elastisch stabile intramedulläre Nagelung (ESIN) eine ideale kindgerechte Osteosyntheseform mit sehr guten funktionellen Ergebnissen. Dies rechtfertigt auch eine primäre ESIN bei repositionspflichtigen Grünholzfrakturen im Sinne einer Narkoseindikation.

\section{Schlüsselwörter}

Kinder - Operation - Osteosynthese .

Narkose $\cdot$ Minimalinvasive Technik

\section{Treatment of pediatric forearm shaft fractures. What can be treated conservatively?}

\section{Abstract}

All forearm fractures with no or only minimal dislocation should still be treated by immobilization with an upper arm plaster cast. In cases of severe dislocation primary operative repositioning including elastic stable intramedullary nailing (ESIN) osteosynthesis is the best form of treatment for children with excellent functional results. In addition, greenstick fractures which need repositioning during general anesthesia should also undergo primary ESIN osteosynthesis.

Keywords

Children - Operation - Osteosynthesis . Anesthesia - Minimally invasive technique

Die Operationsindikationen für dislozierte Unterarmfrakturen sind in der - Tab. 2 aufgezählt.

Neben dem ESIN-Operationsverfahren ist der Fixateur externe für 2. und 3. offene Frakturen und für Mehrfragmentfrakturen indiziert. Bei älteren Kindern kann eine Verplattung bei Vorliegen einer Pseudarthrose oder bei sekundärer 

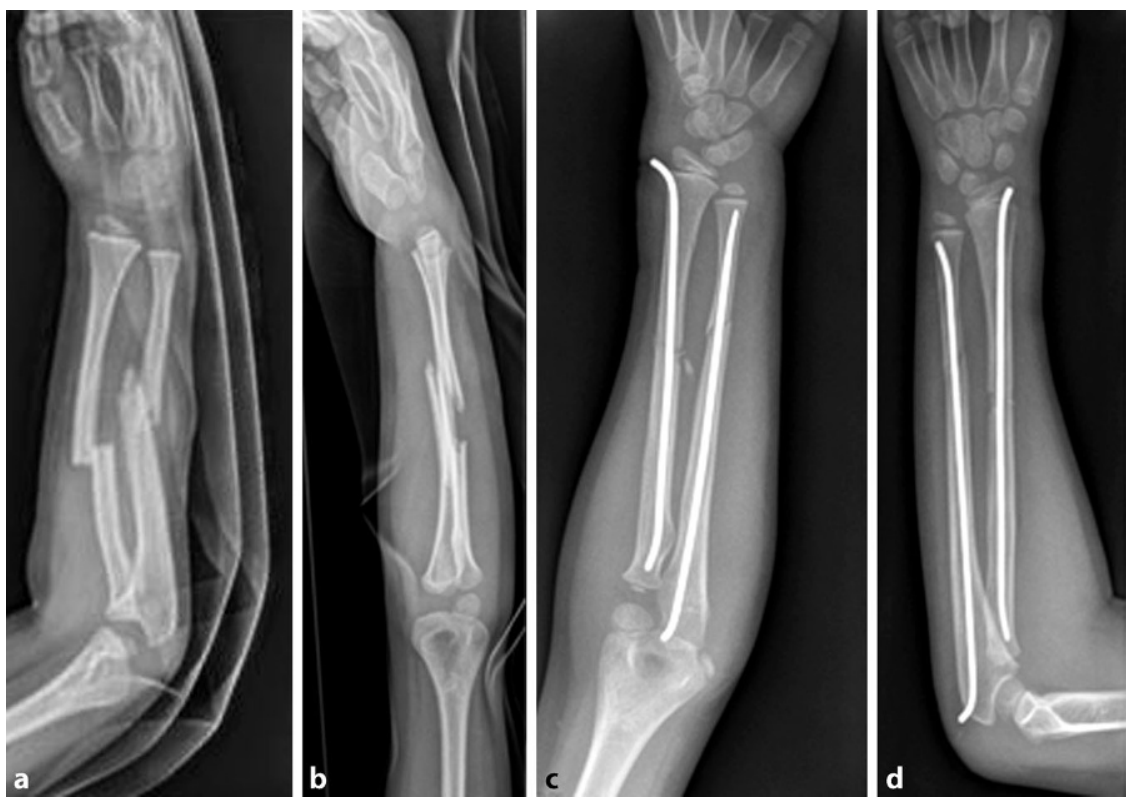

Abb. 4a-d $\Delta$ Fallbeispiel 3: typische instabile verkürzte Unterarmschaftfraktur mit Operationsindikation (13 Jahre, männlich). a, b Unfallröntgenaufnahmen a. p. und seitlich mit erheblicher Dislokation, c, $d$ postoperative Röntgenkontrolle a. p. und seitlich nach ESIN-Osteosynthese
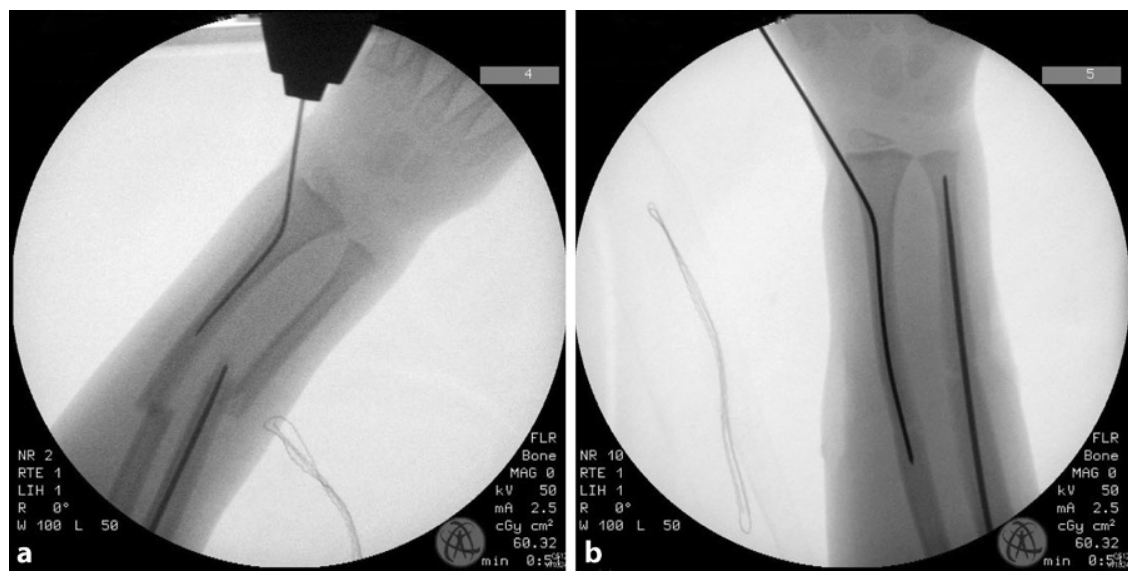

Abb. 5 A Fallbeispiel 4: Repositionshilfe mit bilateraler Prevot-Joystick-Technik (6 Jahre, weiblich). a Intraoperative Vorgehensweise mit diametral eingebrachten Markdrähten ante portas zur geschlossenen Repositionshilfe, $\mathbf{b}$ erfolgreiche ante-portas-Technik mit gegenläufiger Markraumschienung

Frakturkorrektur notwendig werden. In der Praxis der ESIN-Osteosynthese, die oft einfach durchzuführen ist, können jedoch auch Probleme auftreten, die in der Regel mit kleinen Tricks zu lösen sind (• Tab. 3).

Nach erfolgter ESIN-Osteosynthese ist die Fraktur immer übungsstabil und der Unterarm frühzeitig schmerzadaptiert im Alltag benutzbar. Es ist in der Regel kein zusätzlicher Gips indiziert. Sport kann ab der 4. bis 6. Woche ausgeführt werden. Die Materialentfernung ist erst nach 4 bis 6 Monaten je nach Alter zu planen.

Die erste Röntgenkontrolle erfolgt am Operationstag. Um den 28. postoperativen Tag kann die zweite Röntgenkontrolle erfolgen, um eine frühe Sportfreigabe bei erkennbarer Konsolidierung zu gestatten. Die letzte Röntgenkontrolle kann ab dem Tag 90 postoperativ vorgenommen werden, um die Materialentfernung (ME) nach 4 bis 6 Monaten zu indizieren. Refrakturen sind zu 2-5\% möglich, meistens bei zu früher ME. Insgesamt hat die ESIN als bewährtes Verfahren zu
$95 \%$ sehr gute und gute Ausheilungsergebnisse [6].

\section{Fazit für die Praxis}

\section{- Die Behandlung von Frakturen} im Kindesalter ist charakterisiert durch eine differenzierte Therapie mit zunehmender Indikation zur Operation.

- Dabei finden zunehmend minimalinvasive Techniken Anwendung (ESIN) bei möglichst geschlossenem Repositionsmanöver.

- Typisch ist eine frakturferne Implantation des Materials mit möglichst kurzer Liegedauer.

- Für die diaphysäre Unterarmfraktur ist die ESIN eine ideale kindgerechte Osteosyntheseform mit nahezu ausnahmslos funktionell sehr guten Ergebnissen. Dies rechtfertigt aus unserer Sicht eine primäre ESIN ebenfalls bei repositionspflichtigen Grünholzfrakturen (Narkoseindikation).

- Für die Kinder sind eine frühe Mobilisation und funktionelle Behandlung mit schneller sozialer Reintegration von zunehmender Bedeutung.

\section{Korrespondenzadresse}

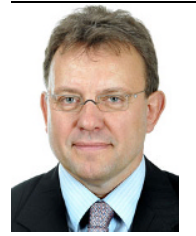

Dr. K. Großer

Klinik für Kinderchirurgie \& Kinderurologie, Zentrum für schwerbrandverletzte Kinder, Frau-Mutter-Kind-Zentrum Erfurt, Helios-Klinikum GmbH Erfurt

Nordhäuser Str. 74, Erfurt,

Deutschland

kay.grosser@

helios-gesundheit.de

\section{Einhaltung ethischer Richtlinien}

Interessenkonflikt. K. Großer gibt an, dass kein Interessenkonflikt besteht.

Dieser Beitrag beinhaltet keine vom Autor durchgeführten Studien an Menschen oder Tieren. Alle Patienten, die über Bildmaterial oder anderweitige Angaben innerhalb des Manuskripts zu identifizieren sind, haben hierzu ihre schriftliche Einwilligung gegeben. Im Falle von nicht mündigen Patienten liegt die Einwilligung eines Erziehungsberechtigten oder des gesetzlich bestellten Betreuers vor. 


\section{Übersichten}

The supplement containing this article is not sponsored by industry.

\section{Literatur}

1. Kraus R, SchneidmüllerD, Roeder C(2005) Haeufigkeit von Frakturen der langen Roehrenknochen im Wachstumsalter.Dtsch Arztebl Int 102:838

2. Herrmann B, Dettmeyer R, Banaschak S, Thyen U (2016) Kindesmisshandlung. Springer, Berlin, S89-106

3. Slongo TF, Audige L, Group AOPC (2007) Fracture and dislocation classification compendium for children: the AO pediatric comprehensive classification for long bone fractures (PCCF). J Orthop Trauma 21:S135-160

4. Weinberg AM, Schneidmüller D (2010) Unfallchirurgie bei Kindern. Deutscher Ärzteverlag, Köln

5. Von Laer L, Hasler C (2000) Spontankorrekturen, Wachstumsstörungen und posttraumatische Deformitäten nach Frakturen im Bereich des Vorderarmes am wachsenden Skelett. Handchir Mikrochir Plast Chir 32:231-241

6. Oberle $M$, Schlickewei W (2011) Unterarm. In: Dietz HG, Illing P, Schmittenbecher PP, Slongo Th, Sommerfeldt DW (Hrsg) Praxis der Kinder- und Jugendtraumatologie. Springer, Berlin, S319-329

7. Von Laer L, Kraus R, Linhardt WE (Hrsg) (2007) Frakturen und Luxationen im Wachstumsalter. Thieme, Stuttgart

8. Marzi J (Hrsg) (2009) Kindertraumatologie. Springer, Berlin, S212-213 